\title{
BMJ Open Exploring cardiovascular health: the Healthy Life in Suriname (HELISUR) study. A protocol of a cross-sectional study
}

Frederieke S Diemer, ${ }^{1,2}$ Jet Q Aartman, ${ }^{2}$ Fares A Karamat, ${ }^{2}$ Sergio M Baldew, ${ }^{3}$ Ameerani V Jarbandhan, ${ }^{3}$ Gert A van Montfrans, ${ }^{4}$ Glenn P Oehlers, ${ }^{1}$ Lizzy M Brewster $2,5,6$

To cite: Diemer FS,

Aartman JQ, Karamat FA, et al. Exploring

cardiovascular health: the Healthy Life in Suriname (HELISUR) study. A protocol of a cross-sectional study. BMJ Open 2014;4:e006380. doi:10.1136/bmjopen-2014006380

- Prepublication history for this paper is available online. To view these files please visit the journal online (http://dx.doi.org/10.1136/ bmjopen-2014-006380).

Received 4 September 2014 Accepted 29 September 2014

CrossMark

For numbered affiliations see end of article.

Correspondence to Frederieke S Diemer; fdiemer@hotmail.com

\section{ABSTRACT}

Introduction: Obesity, hypertension and diabetes are on a dramatic rise in low-income and middle-income countries, and this foretells an overwhelming increase in chronic disease burden from cardiovascular disease. Therefore, rapid action should be taken through preventive population-based programmes. However, in these regions, data on the population distribution of cardiovascular risk factors, and of intermediate and final end points for cardiovascular disease are scarce. The Healthy Life in Suriname (HELISUR) study is a cardiovascular population study in Suriname, which is part of the Caribbean Community. The HELISUR study is dedicated to provide data on risk factors and prevalent cardiovascular disease in the multiethnic population, which is mainly of African and Asian descent.

Methods and analysis: In a cross-sectional, observational population-based setting, a random representative sample of 1800 citizens aged between 18 and 70 years will be selected using a cluster household sampling method. Self-reported demographic, socioeconomic and (cardiovascular) health-related data will be collected. Physical examination will include the assessment of cardiovascular risk factors and prevalent cardiovascular disease. In addition, we will study cardiovascular haemodynamics non-invasively, as a novel intermediate outcome. Finally, fasting blood and overnight urine samples will be collected to monitor cardiometabolic risk factors. The main outcome will be descriptive in reporting the prevalence of risk factors and measures of (sub) clinical end organ damage, stratified for ethnicity and sex-age groups.

Ethics and dissemination: Ethical approval has been obtained from the State Secretary of Health. Data analysis and manuscript submission are scheduled for 2016 . Findings will be disseminated in peer-reviewed journals, and at national, regional and international scientific meetings. Importantly, data will be presented to Surinamese policymakers and healthcare workers, to develop preventive strategies to combat the rapid rise of cardiovascular disease.

\section{Strengths and limitations of this study}

- The Healthy Life in Suriname (HELISUR) study may provide critical data needed for the prevention and control of cardiovascular disease in Suriname. However, the cross-sectional design will limit our inferences to descriptive data.

- The non-invasive cardiovascular haemodynamic assessments are new in low-income or middle-income settings. These assessments may yield vital clues on early changes in the cardiovascular system, which may help prevent premature cardiovascular morbidity and mortality in this high-risk population of predominantly South Asian and African descent.

- Our methods and results may prove to be of use to other countries with similar socioeconomic and population profiles, in the design of strategies to collect data on cardiovascular population health.

\section{BACKGROUND}

Cardiovascular disease (CVD) is rapidly increasing in low-income and middle-income countries (LMIG). ${ }^{1}$ At present, the number of cardiovascular deaths is responsible for the greatest proportion of the overall burden of disease, and projections on mortality attributable to CVD forecast even higher death rates in 2030 (figure 1)..$^{2}$ Awareness is growing that preventive action based on country-specific risk factor data is imperative to combat this burgeoning epidemic. ${ }^{3}$

Suriname is a middle-income country located in the northeastern region of South America (figure 2). The country is populated by different ethnic groups, including people of South Asian, African, Indonesian and Chinese origin, as well as a relatively small indigenous population of Amerindians. As in other $\mathrm{LMIC}$, CVD is a rapidly rising cause of 

by World Bank's Income Categories. The number of projected deaths in millions due to cardiovascular disease (y-axis) is depicted in high-income, middle-income and low-income countries for 2004, 2015 and 2030 (x-axis). ${ }^{2}$ This figure indicates that the projected increase in mortality is the highest in middle-income countries such as Suriname.
Figure 1 Cardiovascular Death

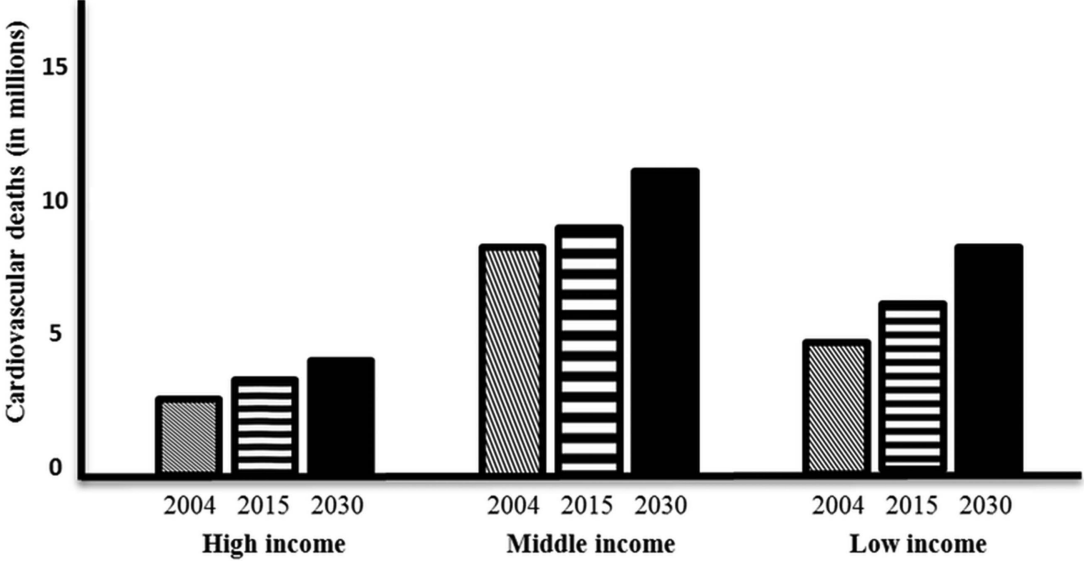

premature mortality. ${ }^{4}$ However, akin to other countries with restrained resources, there is a paucity of data on the population distribution of risk factors, and of intermediate and final end points for CVD.

Taking the projections of a greater increase of CVD mortality in LMIC into account, we designed the Healthy Life in Suriname (HELISUR) study, a population-based study on cardiovascular health among different ethnic groups in an urban setting. The study aims to generate population data on established risk factors for CVD, such as obesity, hypertension, diabetes and hyperlipidaemia. In addition, we will collect data on intermediate end points, including non-invasive haemodynamics, and the prevalence of clinical cardiovascular end organ damage.

\section{METHODS AND ANALYSIS}

\section{Study design}

HELISUR is a cross-sectional multiethnic populationbased study of adults aged between 18-70 years and living in Suriname. The study is scheduled from November 2013 to December 2016. Data analysis and manuscript submission are foreseen from 2016.

\section{Study objectives}

The primary objective is to assess risk factors for CVD and prevalent CVD across sex, age and ethnic groups in the general population of Suriname. Secondary objectives are assessment of ethnic disparities in health behaviour (eg, physical activity, nutrition) and cardiovascular morbidity. Furthermore, we will address the question to what extent established and novel risk factors can explain current CVD. These novel risk factors may include haemodynamic factors, migration to urban areas or novel biochemical risk factors.

\section{Study area}

Suriname is estimated to have 534500 citizens, with $75 \%$ of the population members younger than 45 years of age. $^{5}$ The current mean life expectancy at birth is 71 years. $^{6}$ The vast majority of the population $(70 \%)$ lives in urban areas, mainly the coastal area where the capital, Paramaribo, is located. Table 1 shows an overview of World Development Indicators, indicating the categorisation of Suriname in LMIC.

\section{Study population}

Non-institutionalised participants living in urban areas, aged 18-70 years, will be randomly sampled through the cluster household sampling method, as described in the 'Sampling Scheme'. Pregnant women can be included but will be invited for physical examination 3 months after delivery. Participants who are unable to give informed consent or are deceased before the questionnaire is filled out will be excluded.

\section{Sampling scheme}

Using the methods described by Lwanga et $a l^{7}$ the minimum necessary representative sample size was calculated to be 1092 participants, with a statistical precision of at least 0.01 to estimate the risk factor prevalence at a 95\% confidence level. The sample size is weighted to account for the complex sampling design. Furthermore, we estimated a $40-50 \%$ refusal or dropout rate, based on a literature review of population studies. Therefore, we decided to include 1800 participants.

Household sampling will be used, and households will be selected by randomisation of geographical areas, taking the residence of different ethnic groups into account. This will ensure that the different ethnic groups are adequately represented in the sample. Paramaribo is divided into 1200 enumeration areas out of which 18 will be randomly selected by the General Bureau of Statistics in Suriname. In order to include 1800 participants, 100 individuals will be needed per enumeration area. A group of trained interviewers will each cover one enumeration area, visiting every house and all household members until 100 persons are included, meeting the inclusion criteria. To reduce inclusion bias, if household members are not at home, interviewers will revisit until every household member is included or has stated a refusal to participate. Participants will be invited thereafter for physical examination at the Academic Hospital in Paramaribo. 


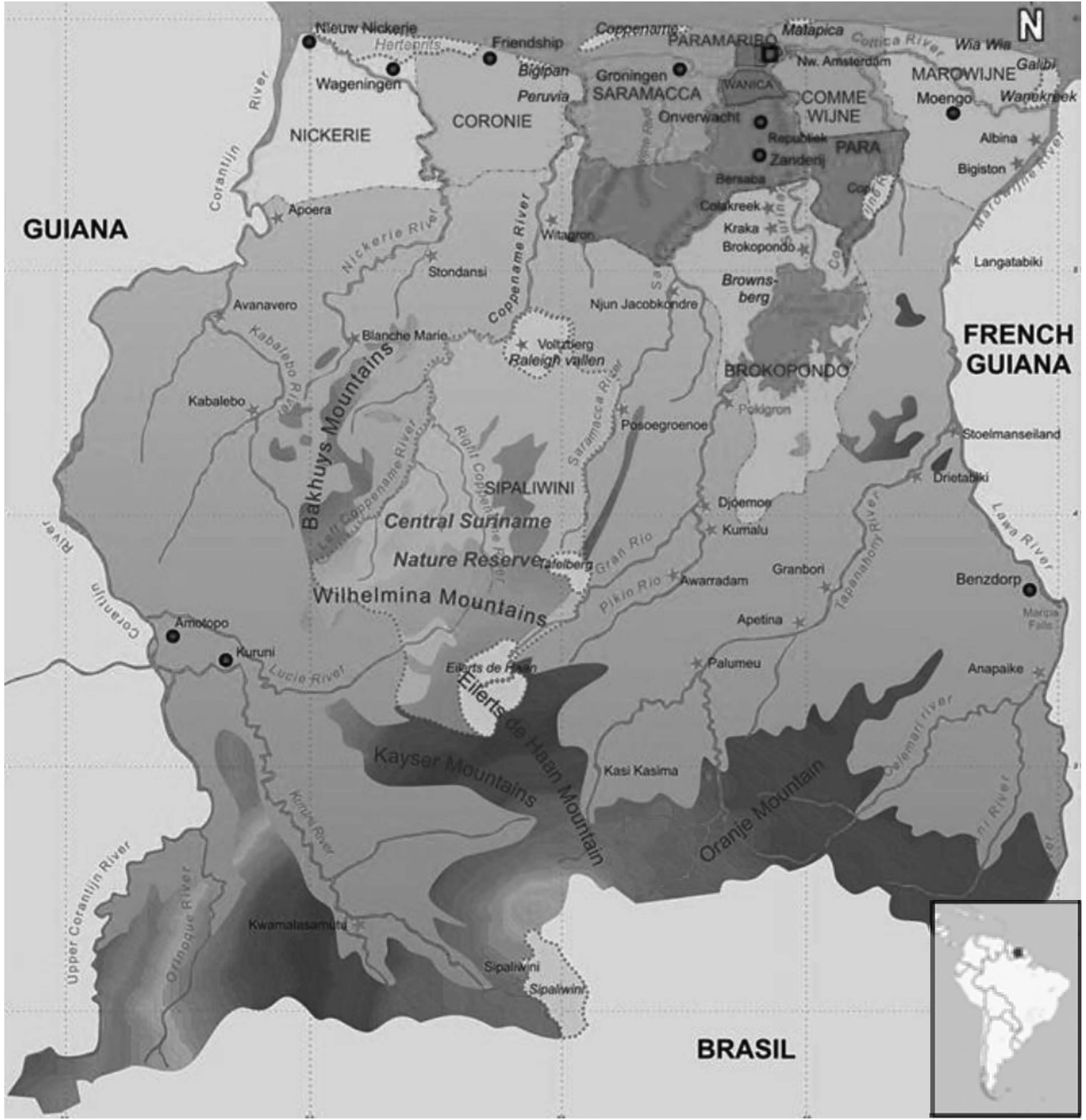

Figure 2 Map of Suriname, South America. Suriname is located in the northeastern part of South America. The country is bordered by French Guiana, Guiana and Brazil, but culturally, economically, and demographically it is part of the Caribbean Community. Urban areas include the capital Paramaribo in northern Suriname and the surrounding suburbs.

Techniques to minimise no-shows include telephone and text message prompting.

\section{Research procedures}

Questionnaire

Participants will fill out a questionnaire at home with the support of a trained interviewer (table 2).

At the day of the physical examination, the questionnaire is updated to match the day of the physical examination and ensure validity of the answers.

\section{Physical examination}

Participants will visit the hospital for a physical examination including anthropometrics and blood and urine tests (table 3). Resting brachial systolic and diastolic blood pressure (SBP and DBP) will be measured twice in the sitting position, with the occluding cuff at the heart level (WatchBP Home; Microlife A.G., Widnau, Switzerland). The ankle-brachial index will be assessed in the supine position by WatchBP Office ABI (Microlife A.G., Widnau, Switzerland), twice on both sides. Finally, a 12-lead electrocardiogram will be recorded (ECG-1200 Biocare; Collateral Medical, Mumbai, India).

Safar ${ }^{8}$ proposed that although the cyclic blood pressure curve is usually described exclusively as SBP and DBP, both dominating the basis of cardiovascular hypertensive epidemiology, the entire blood pressure curve should be taken into consideration to adequately assess cardiovascular damage. Pulsatile arterial haemodynamics are able to predict CVD in essential hypertension, renal 
Table 1 Overview of world development indicators in Suriname

\begin{tabular}{ll}
\hline Parameter & Estimate \\
\hline Income level & Upper middle-income \\
Population & 534500 \\
Life expectancy at birth, years & 71 \\
GNI per capita, \$ & 8480 \\
GDP, \$ & $4.7 \times 10^{15}$ \\
GDP growth, \% & 4.5 \\
Inflation, \% & 5.0 \\
\hline
\end{tabular}

World development indicators estimated by the World Bank. ${ }^{6}$ Data on mean life expectancy at birth are from 2011; all other data are from 2012.

GDP, gross domestic product; GNI, gross national income.

failure, diabetes mellitus and ageing. Therefore, we will assess pulsatile arterial haemodynamics non-invasively. These novel indices in cardiovascular population risk factor assessment include central systolic pressure, central pulse pressure, augmentation index and pulse wave velocity, as measured and calculated by the Arteriograph (TensioMed, Budapest, Hungary). In addition, we will estimate the cardiac output, left ventricular contractility and systemic vascular resistance, by means of continuous non-invasive finger arterial pressure using the Nexfin HD Monitor (BMEYE, Amsterdam, The Netherlands). Participants will receive an annotated summary of the main results.

\section{Handling and storage of data and documents}

All data will be handled confidentially and anonymously. A participant identification code list will be used to link the data to the participant. This code will not be based on the patients' initials and/or date of birth. The key to the code will be saved separately from the database and will be kept by the primary investigator and the data manager. The handling of personal data will comply with the Personal Data Protection Act. With the participant's permission, biological samples will be stored during 15 years, annotated with an identification code.

\section{Statistical methods}

The primary outcome is descriptive: the prevalence of cardiovascular risk factors will be provided, stratified for ethnicity, sex, age, socioeconomic status and/or other classifications of interest. Means (continuous variables) or percentages (categorical variables) of risk factors (including biochemical factors) and potential confounders will be reported, classified for ethnicity, sex, age and/or other classifications of interest. To study associations of potential risk factors or determinants with (intermediate) disease outcomes, linear (for continuous outcomes) or logistic regression analyses (for dichotomous outcomes) will be performed.

\section{Risk assessment}

The overall cardiovascular risk will be estimated by the Framingham Risk Score for CVD (10-year risk). Logarithms of age, serum lipids, SBP, smoking and diabetes mellitus will be used. ${ }^{9}$ The Rose angina questionnaire will be used to diagnose angina pectoris and classify participants into definite angina, possible and non-exertional chest pain. ${ }^{10}$ The medical outcome survey Short Form-12 is validated as a quality of life assessment tool for the general population. ${ }^{11}$ Physical activity will be assessed by means of the International Physical Activity Questionnaire, which classifies participants into three levels of physical activity (low, moderate or vigorous). ${ }^{12}$

\section{Quality control}

We will ensure that quality controls will be executed throughout the conduct of the study, with regard to

Table 2 Health indicators in the HELISUR questionnaire

\begin{tabular}{ll}
\hline Theme & Explanatory factors \\
\hline General & Demographic factors \\
& Sex, age, marital status, household composition, urban status, educational level, occupational status \\
& Ethnicity \\
& Self-reported ethnicity of participant and (grand) parents \\
& Proximal risk factors \\
& Health-related behaviour: smoking, alcohol intake, salt intake, dietary pattern, physical activity, weight \\
& perception; \\
& Healthcare use and related factors: therapy compliance, GP quality perception; \\
& Working conditions: physical activity at work \\
& History of high-blood pressure, diabetes, hypercholesterolaemia, fainting, or venous thrombosis; \\
& Symptoms or history of end organ damage of heart or vessels \\
Cardiovascular & (angina pectoris, myocardial infarction, claudicatio intermittens with the Rose questionnaire; and \\
health & neurological or transient neurological attacks); \\
& Family history of high-blood pressure, diabetes, hypercholesterolaemia, cardiovascular disease or \\
& sudden death
\end{tabular}

Overview of health indicators to be explored in the HELISUR questionnaire. The questionnaire addresses demographic factors, as well as factors concerning cardiovascular health, including the presence of cardiovascular risk factors and cardiovascular disease. GP, general practitioner. 
Table 3 Physical examination and laboratory analyses

\begin{tabular}{ll}
\hline Study procedure & Variables \\
\hline \begin{tabular}{ll} 
Physical & Anthropometry: height, weight, waist-hip circumference, thigh, arm and calf circumference \\
examination & Heart function measured by Nexfin: non-invasive measurement of cardiac output, stroke volume and \\
& systemic vascular resistance \\
& Arterial stiffness measured by an Arteriograph: non-invasive measurement of pulse wave velocity, \\
& augmentation index and central blood pressure \\
& Ankle-brachial index: peripheral vascular disease \\
& Sitting blood pressure \\
& Electrocardiogram: left ventricular hypertrophy \\
& Collection of fasting blood sample and overnight urine sample \\
& Blood: haemoglobin, HbA1c, glucose, triglycerides, total cholesterol, HDL cholesterol, LDL cholesterol, \\
Laboratory & Creatine kinase \\
analyses & Urine: pH, glucose, microalbuminuria \\
\hline Overview of the cardiometabolic risk assessment with physical and laboratory examination, including non-invasive cardiovascular \\
haemodynamics.
\end{tabular} \\
HbA1c, glycated haemoglobin; HDL, high-density lipoprotein; LDL, low-density lipoprotein.
\end{tabular}

sample selection, data collection, data processing and reporting. The well-defined sampling design and prior estimation of sample size reduces sampling errors. Furthermore, we tested and validated the questionnaire, and included multiple cross-checked questions on the same topic to validate the results. Additionally, healthcare workers who collect the data will be well trained according to standard operating procedures. On every day of the data collection, we monitor and ascertain the performance of our measurement devices and check the questionnaire of the participants for answer's correctness and completeness. Our database allows range checks for accuracy and checks for completeness of the entered data. We used independent double data entry followed by matching and checking for data entry errors. Data cleaning will be performed according to expert consensus. Finally, we will check internal and external consistency of the analysed data before writing reports. ${ }^{13}$

\section{Ethical considerations}

This study will be conducted according to the principles of the Declaration of Helsinki (59th WMA General Assembly, Seoul, October 2008) and in accordance with the Medical Research Involving Human Subjects Act (WMO). Participants will be required to give informed consent for the study participation, which includes the permission to store a blood and urine sample for future research; to link registries containing data relating to the participant's health (eg, pharmacy, hospital, general practitioner); and finally to link with mortality registries where applicable.

\section{DISCUSSION}

CVD is generally viewed as a disease of affluent countries. However, it has become the main cause of disease burden and premature death in low-income and middle-income countries, killing more people than malaria, AIDS and tuberculosis together. ${ }^{1}$ The rapid increase in urbanisation in these countries creates lifestyle changes, with greater consumption of fat, salt and sugar, and less physical exercise. In line with these changes, health projections suggest that CVD burden will increase even further in these regions (from $33 \%$ in 2002 to $45 \%$ in 2030), with potentially devastating effects on personal and family incomes, and on the national economy. ${ }^{2}$

With this in mind, we designed the HELISUR population study to gather data on the age distribution and prevalence of risk factors for cardiovascular disease, and of CVD morbidity and mortality. To the best of our knowledge, for the first time in this setting, we will include detailed assessments of non-invasively measured cardiovascular haemodynamic parameters such as aortic pulse wave velocity and central aortic blood pressure as intermediate outcomes for cardiovascular disease. These data are expected to be of great value to further probe the cardiovascular health status of the participants.

Limitations pertain to the cross-sectional design of the study, permitting only descriptive outcomes. Furthermore, we need to be wary of selective survival bias, as any risk factor that results in death might be under-represented. Importantly, the HELISUR study will yield extensive cardiovascular data, much needed by healthcare workers and policymakers to design measures to prevent the tsunami of CVD expected within the coming decades.

\section{Author affiliations}

${ }^{1}$ Department of Cardiology, Academic Hospital Paramaribo, Paramaribo, Suriname

${ }^{2}$ Department of Vascular Medicine, Academic Medical Center, Amsterdam, The Netherlands

${ }^{3}$ Department of Physiotherapy, Anton de Kom University of Suriname,

Paramaribo, Suriname

${ }^{4}$ Department of Internal Medicine, Academic Medical Center, Amsterdam, The Netherlands

${ }^{5}$ Department of Public Health, Anton de Kom University of Suriname, Paramaribo, Suriname

${ }^{6}$ Department of Social Medicine, Academic Medical Center, Amsterdam, The Netherlands 
Contributors GPO, GAvM and LMB conceived the study, wrote the protocol and obtained funding. GPO sought ethical approval. FSD and LMB drafted the manuscript. All authors read, edited draft versions and approved the final manuscript.

Competing interests None.

Patient consent Obtained.

Ethics approval The study was reviewed and approved by the Ethics Committee of the Ministry of Health in Suriname (dated 10 October 2012).

Provenance and peer review Not commissioned; externally peer reviewed.

Open Access This is an Open Access article distributed in accordance with the Creative Commons Attribution Non Commercial (CC BY-NC 4.0) license, which permits others to distribute, remix, adapt, build upon this work noncommercially, and license their derivative works on different terms, provided the original work is properly cited and the use is non-commercial. See: http:// creativecommons.org/licenses/by-nc/4.0/

\section{REFERENCES}

1. Global status report on non-communicable diseases 2010. Geneva, Switzerland: World Health Organization, 2011. http://www.who.int/ $\mathrm{nmh} /$ publications/ncd_report_full_en.pdf (accessed 6 Oct 2014).

2. Mathers CD, Loncar D. Projections of global mortality and burden of disease from 2002 to 2030. PLoS Med 2006;3:2011-30.

3. Report of the Caribbean Commission on Health and Development Kingston, Jamaica: Pan American Health Organization and the Caribbean Community, 2006. http://www.who.int/macrohealth/action/ PAHO_Report.pdf (accessed 6 Oct 2014).
4. Punwasi W. Doodsoorzaken Suriname 2010-2011. Paramaribo, Suirname: Ministry of Health, Bureau Public Health, 2011. http:// www.bogsur.sr/index.php/ct-menu-item-11/11-doodsoorzaken-insuriname-2010-2011.pdf (accessed 6 Oct 2014).

5. General Bureau of Statistics. Voorlopige resultaten uit de 8ste volksen woningtelling in Suriname. Paramaribo, Suriname: Algemeen Bureau voor de Statistiek, 2012. https://unstats.un.org/unsd/ demographic/sources/census/2010_PHC/Suriname/SURCensus2012-vol1.pdf (accessed 6 Oct 2014).

6. The World Bank Group. Suriname, 2014. http://data.worldbank.org/ country/suriname (accessed 6 Oct 2014).

7. Lwanga SK, Lemeshow S. Sample size determination in health studies: a practical manual. Geneva, Switzerland: World Health Organization, 1991:1-3. http://whqlibdoc.who.int/publications/ 9241544058 (p1-p22).pdf (accessed 6 Oct 2014).

8. Safar ME. Pulse pressure, arterial stiffness and wave reflections (augmentation index) as cardiovascular risk factors in hypertension. Ther Adv Cardiovasc Dis 2008;2:13-24.

9. D'Agostino RB Sr, Vasan RS, Pencina MJ, et al. General cardiovascular risk profile for use in primary care: the Framingham Heart Study. Circulation 2008;117:743-53.

10. Rose GA. The diagnosis of ischaemic heart pain and intermittent claudication in field surveys. Bull WHO 1962;27:654-8.

11. Ware JE, Kosinski M, Keller SD. A 12-item short-form health survey: construction of scales and preliminary tests of reliability and validity. Med Care 1996;34:220-33.

12. Craig $\mathrm{CL}$, Marshall AL, Sjostrom M, et al. International physical activity questionnaire: 12 -country reliability and validity. Med Sci Sports Exerc 2003;35:1381-95.

13. Alkerwi A, Sauvageot N, Donneau AF, et al. First nationwide survey on cardiovascular risk factors in Grand-Duchy of Luxembourg (ORISCAV-LUX). BMC Public Health 2010;10: $468-9$. 